\title{
Structure-Transport Properties of Topological Nanowires
}

\author{
Judy Cha
}

Yale University, United States

Since the discovery of the two-dimensional topological insulating phase in mercury telluride quantum wells in 2007 [1], the number of topological materials that have been experimentally verified have steadily increased. About a quarter of all known materials are now predicted to be topological in nature [2], spanning from topological metals to topological superconductors, whose topological properties can enable a host of potentially transformative electronic applications. In particular, topological nanowires are important for investigating Majorana bound states expected at the ends of topological superconducting nanowires for quantum computing [3] and for electron scattering mechanisms for low-resistance interconnect applications.

For topological nanowires, the dimensions, morphology, and the crystallinity of the nanowires critically affect the transport properties of the nanowires. For example, in topological crystalline insulator SnTe nanowires, we have shown that the ferroelectric phase transition temperature changes systematically with the diameter of the nanowire [4]. In addition, the microstructure of the domain boundaries between two ferroelectric domains in SnTe nanowires is believed to affect the nature of electron-electron interactions when superconductivity is induced in SnTe nanowires [5]. In the case of topological metal MoP nanowires [6], the resistivity of the nanowires is found to be diameter-dependent: surprisingly, the resistivity decreases with decreasing diameter of MoP nanowires. As the electron transport of MoP is anisotropic [7], a detailed understanding of the microstructure of MoP nanowires is needed in order to realize a very-low resistance interconnect.

Atomic-resolution structural characterizations of these topological nanowires by transmission electron microscopy (TEM) and in situ cryogenic TEM are thus critical to harness the full potential of the topological properties for electronic applications. In this talk, I will highlight the structure-transport property relationships of the aforementioned SnTe and MoP nanowires and in situ cryo-TEM studies of the phase transition of SnTe nanowires. Specifically, I will present the microstructural evolution of these nanowires as a function of diameter and their effects on the transport properties. If time allows, I will also discuss phase transitions of two-dimensional topological materials, such as MoTe2 and WTe2.

Acknowledgements: The work on SnTe nanowires is funded by DOE DE-SC0014476 and the work on MoP nanowires by SRC nCORE IMPACT. J. J. C. gratefully acknowledges support from the Gordon and Betty Moore Foundation EPiQS Synthesis Award.

\section{References}

[1] M. Konig, S. Wiedmann, C. Brune, A. Roth, H. Buhmann, L. W. Molenkamp, X.-L. Qi, S.-C. Zhang, Science 318, p.766-770 (2007).

[2] M. G. Vergniory, L. Elcoro, C. Felser, N. Regnault, B. A. Bernevig, Z. Wang, Nature 566, 480-485 (2019).

[3] M. Leijnse and K. Flensberg, Semicond. Sci. Technol. 27, 124003 (2012).

[4] P. Liu, H. J. Han, J. Wei, D. J. Hynek, J. L. Hart, M. G. Han, C. J. Trimble, J. R. Williams, Y. Zhu, J. J. Cha, ACS Applied Electronic Materials 3, p.184-191 (2020). 
[5] C. J. Trimble, M. T. Wei, N. F. Q. Yuan, S. S. Kalantre, P. Liu, J. J. Cha, L. Fu, J. R. Williams, arXiv: 1907.04199 (2020).

[6] H. J. Han, D. Hynek, Z. Wu, P. Liu, J. V. Pondick, S. Yazdani, J. M. Woods, M. Yarali, Y. Xie, H. Wang, J. J. Cha, APL Materials 8, 011103 (2020).

[7] N. Kumar et. al., Nature Communications 10:2475 (2019). 